\title{
The Use of Opioids for Breakthrough Pain in Acute Palliative Care Unit by Using Doses Proportional to Opioid Basal Regimen
}

\author{
Sebastiano Mercadante, MD, ${ }^{*}+$ Patrizia Villari, MD, ${ }^{*}$ Patrizia Ferrera, MD, BS,* \\ Salvatore Mangione, MD, $\dagger$ and Alessandra Casuccio, BS $\ddagger$
}

\begin{abstract}
Objectives: To determine the efficacy and safety of different opioids used in doses proportional to the basal opioid regimen for the management of breakthrough pain (BP).
\end{abstract}

\begin{abstract}
Methods: In 66 patients consecutive patients admitted to a pain relief and palliative care unit, the efficacy and safety of different opioids used in doses proportional to the basal opioid regimen for the management of breakthrough pain (BP) were assessed. The choice of the opioid to be administered as rescue medication was based on the characteristics of patients, clinical stability, compliance, preference, and so on. For each episode, nurses were instructed to routinely collect changes in pain intensity and emerging problems when pain became severe (T0), and to re-assess the patient 15 minutes after the opioid given as a rescue medication (T15).
\end{abstract}

Results: Six hundred twenty four episodes of BP were recorded during admission. Intravenous morphine (IV-MO) and oral transmucosal fentanyl (OTFC) were most frequently administered. Of 503 events available, 427 episodes were defined as successfully treated, while 76 episodes required a further administration of opioids. Pain intensity significantly decreased at T15 in all the groups $(P<0.001)$. In $97.2 \%$ and $90.7 \%$ of cases treated with IV$\mathrm{MO}, \mathrm{BP}$ events had a reduction in pain intensity of more than $33 \%$ and $50 \%$, respectively. In $99.2 \%$ and $97.6 \%$ patients receiving OTFC, BP events had a reduction in pain intensity of more than $33 \%$ and $50 \%$, respectively.

Discussion: This survey suggests that doses of opioids for BP proportional to the basal opioid regimen, are very effective and safe in clinical practice, regardless the opioid and modality used.

Key Words: cancer pain, breakthrough-episodic pain, opioids

(Clin J Pain 2010;26:306-309)

In the cancer population, breakthrough pain (BTP) is a transitory exacerbation of the pain superimposed on an otherwise stable pain pattern in patients treated with opioids. ${ }^{1}$ BTP is normally severe in intensity and has a rapid onset. The presence of BTP has been considered as a negative prognostic factor that interfered with the quality of life of the patients. ${ }^{2}$ Availability of supplemental doses of

Received for publication May 15, 2009; revised September 14, 2009; accepted September 19, 2009.

From the *Anesthesia and Intensive Care Unit \& Pain Relief and Palliative Care Unit, La Maddalena Cancer Center, Palermo, Italy; $\dagger$ Intensive Care and Emergency, Palliative Medicine Teaching, Palermo, Italy; and $\ddagger$ University of Palermo, Palermo, Italy.

Reprints: Sebastiano Mercadante, MD, Anesthesia and Intensive Care Unit and Pain Relief and Palliative Care Unit, La Maddalena Cancer Center, Via S.Lorenzo 312, 90145 Palermo, Italy (e-mail: terapiadeldolore@lamaddalenanet.it).

Copyright (C) 2010 by Lippincott Williams \& Wilkins opioids (rescue medication) in addition to the continuous analgesic medication is the main treatment suggested to manage these pain flares, either during dose titration or when basal pain is under control. Current dosing recommendations for BTP generally suggest that the effective dose of BTP medication must be a percentage of a patient's total daily opioid dose. ${ }^{3}$ These recommendations, which are based entirely on anecdotal experience, favor the selection of a short-acting opioid at a dose proportional to the total daily dose. As the pain relief is usually required urgently, routes of administration designed to delivery drugs rapidly are often chosen. A short onset of the effect is commonly obtainable only with parenteral or transmucosal administration of opioid analgesics.

In preliminary experiences, intravenous morphine (IV$\mathrm{MO}$ ) proved to be safe and effective for the management of $\mathrm{BTP}^{4,5}$ and has been the preferred treatment in the last 5 years in our acute pain relief and palliative care unit. In studies of the oral transmucosal fentanyl citrate (OTFC), ${ }^{6-9}$ the anecdotal assumption that the effective dose as needed is a percentage of the opioid daily dose was contradicted by the observation that the OTFC dose needs to be titrated, introducing a new variable in choosing the doses to be administered, which could reduce patient's and physician's compliance. The need for titration may discourage patients, particularly outpatients or home care patients, to continue titration in daily activity. In an earlier open controlled study, both IV-MO and OTFC in doses proportional to the scheduled daily dose of opioids were safe and effective in patients experiencing pain exacerbation, ${ }^{10}$ suggesting that opioids given as needed, including OTFC, could be safely used at proportional doses, regardless of the modality of administration. Finally, indirect observations from data pooled from trials of OTFC showed a relationship between the BTP dose and around-the-clock dose, despite large variability in patients' dose requirements. ${ }^{11}$ The aim of this audit was to confirm the safety of such an approach with the available formulations of opioids commonly used for treating BTP in our country, avoiding the process of dose titration for BTP in a large number of patients assessed in a real clinical scenario representing the routine activity of an acute palliative care unit.

\section{METHODS}

All the patients consecutively admitted to a Pain Relief and Palliative Care unit in a period of 6 months from January 2008 to June 2008 were surveyed. From this sample, patients who were receiving opioids in doses of oral morphine equivalents equal to or more than $60 \mathrm{mg}$ daily, 
who were prescribed opioids for BTP of different nature, were included into the study. Informed consent for recording data for the study from all patients and institutional approval were obtained.

Patients were treated according to the department policy. After establishing around-the-clock opioid medication, according to opioid titration process, patients were encouraged to call when their pain got severe either during the titration phase or when basal pain was controlled (considered when the mean pain intensity is equal or less than 4/10) and superimposed episodes of BTP occur.

The choice of opioids for basal pain and BTP was based on clinical judgment, according to different factors, including the patient's characteristics, clinical needs, patients' compliance, opioid used for background analgesia, and preference. Department's policy suggests that the dose to be given was proportional to the dose administered for background analgesia, $1 / 5$ of the oral morphine equivalent to daily doses, taking into account also the route conversion ratios. ${ }^{4}$ For example, a daily oral morphine dose $60 \mathrm{mg}$ corresponds to an intravenous dose of $20 \mathrm{mg}$ ( $1 / 3 \mathrm{ratio})$, and then is converted to $4 \mathrm{mg}$ of the intravenous morphine $(1 / 5)$ as the dose for BTP. Similarly, oral morphine (OR-MO) is $12 \mathrm{mg}$ and OTFC is $200 \mu \mathrm{g}$. Doses of the other opioids, oral oxycodone (OR-OX), oral methadone (OR-ME), and intravenous methadone (IV-ME), also used for treating BTP, were adapted using conversion ratios routinely adopted by the unit (Table 1). ${ }^{4}$ The opioid dose is modified according to eventual changes of the daily oral morphine dosage. Written orders for BTP, including drugs and doses to be administered when pain gets severe enough are routinely given in the therapy chart. For each episode, trained nurses recorded pain intensity (numerical scale 0 to 10) and adverse effects severe enough in intensity to require medical intervention, when called for pain increases considered to be severe in intensity by patients (T0), and 15 minutes after administering the rescue dose of opioids (T15). ${ }^{5}$ The administration of BTP opioid medication was considered unsuccessful whenever a further BTP medication was required in the subsequent 2 hours. As a routine, a physician on duty is present in the department, and the palliative care team is available on call for any emergency or consultation in case of development of severe adverse effects. The principal outcome was the evaluation of the number of patients who could benefit from opioids given as rescue medication within 15 minutes, a clinically meaningful time to evaluate a dose administered as needed, using proportional doses of the basal opioid dosage, regardless of the

TABLE 1. Conversion Ratios Conventionally Used in the Unit

\begin{tabular}{lc}
\hline & Dose $(\mathbf{m g})$ \\
\hline IV-MO & 33 \\
OR-MO & 100 \\
TTS fentanyl & 1 \\
IV-ME & 16 \\
OR-ME & 20 \\
OR-OX & 70 \\
\hline
\end{tabular}

The opioid dose for BTP is $1 / 5$ of the oral morphine equivalent daily doses, also taking into account route conversion ratios (see example in Methods). ${ }^{4}$

IV-ME indicates intravenous methadone; IV-MO, intravenous morphine; OR-ME, oral methadone; OR-MO, oral morphine; OR-OX, oral oxycodone; TTS-fentanyl, transdermal fentanyl. opioid and modality used and the occurrence of adverse effects severe enough in intensity to require medical intervention.

\section{Statistical Analysis}

Patients were divided according to the age: $\leq 65$ years and $>65$ years. Frequency analysis was carried out using the $\chi^{2}$ test. Frequency analysis was carried out using the Pearson chi-square test and Fisher exact test. The 1-way analysis of variance and Kruskal-Wallis Statistic test were used to compare the different parametric or nonparametric variables. Data were analyzed by SPSS Software 14.0 version (SPSS, Inc., Chicago, Ill, US). All $P$-values were 2 sided, and $P$ values less than 0.05 were considered statistically significant.

\section{RESULTS}

Sixty-six patients were surveyed. The mean age was $66.7( \pm 12.2)$, and $39(59.1 \%)$ of the patients were over 65 years. Forty-two patients were male. Opioids used for background analgesia were in a rank order: buprenorphine (18), fentanyl (16), IV-MO (11), OR-OX (10), OR-MO (6), hydromorphone (4), and OR-ME (1). Six hundred twentyfour episodes of BTP were recorded during admission $(5.3 \mathrm{~d}, \mathrm{SD} \pm 3.6)$. Each patient presented a mean of 8.7 $(\mathrm{SD} \pm 8)$ episodes during admission (median 2 episodes/d).

IV-MO and OTFC were most frequently administered. Three hundred eighty-six, 152, 42, 5, 32, and 7 episodes were treated with IV-MO, OTFC, OR-MO, OR-ME, IV-ME, and OR-OX, respectively. In 121 events, documentation regarding changes in pain intensity was incomplete in the record sheet ( 67 and 54 events were incomplete at $\mathrm{T} 0$ and $\mathrm{T} 15$, respectively). Of the 503 valuable episodes, 427 were successful treated without any further request, whereas 76 episodes $(15.1 \%)$ required a further administration of opioids within the subsequent 2 hours.

Pain intensity significantly decreased at T15 in all the groups, $P \leq 0.001$ (Table 2), regardless of the drug and modality used. In $241(97.2 \%)$ and $225(90.7 \%)$ episodes occurring in patients receiving IV-MO, BTP events had a reduction in pain intensity of more than $33 \%$ and $50 \%$, respectively. In $123(99.2 \%)$ and $121(97.6 \%)$ of episodes occurring in patients receiving OTFC, BTP events had a reduction in pain intensity of more than $33 \%$ and $50 \%$, respectively. Data regarding other opioids are listed in Table 3. Cumulative percentages of responders are reported in Figure 1.

No differences in gender and age and percentage changes of pain intensity were observed $(P=0.383$ and $P=0.788$, for $33 \%$ and $50 \%$ of pain reduction, respectively, for gender, and $P=0.288$ and $P=0.218$, respectively, for age). No adverse effects, severe enough in intensity to require medical intervention, were observed.

\section{DISCUSSION}

The opioid dose to be administered for BTP is still controversial. Owing to the variability in the presentation of pain flares, it is difficult to provide clear guidelines. ${ }^{12}$ Dosing recommendations for morphine have been based on anecdotal experience; they suggest that the effective dose of BTP medication is a percentage of the patient's total daily opioid dose. EAPC recommendations suggest $1 / 6(17 \%)$ of the daily dose as a starting point. ${ }^{3}$ On the other hand, all the trials with OTFC have contradicted this anecdotal 


\begin{tabular}{lcccccc}
\hline \multicolumn{7}{l}{ TABLE 2. Doses and Changes in Pain Intensity in Patients Treated With Different Opioids for BTP Successfully (See Text) } \\
\hline & IV-MO & OTFC & OR-MO & IV-ME & OS-ME & OR-OX \\
\hline Evaluable episodes & 248 & 124 & 31 & 15 & 4 & 5 \\
Dose (SD) & $14.9(17.9)$ & $637(432)$ & $7.5(2.3)$ & $5.87(2.96)$ & $10(0)$ & $7.75(1.7)$ \\
T0 Pain intensity & $7.2(0.96)$ & $6.9(0.74)$ & $6.7(0.9)$ & $6.9(1)$ & $7.14(0.9)$ \\
T15 & $2.72(0.9)$ & $2.53(0.75)$ & $2.39(0.66)$ & $2.86(0.6)$ & $3.5(1.3)$ & $3.4(3.4)$ \\
$P$ & 0.0005 & 0.0005 & 0.0005 & 0.0005 & 0.003 & 0.001 \\
\hline
\end{tabular}

IV-ME indicates intravenous methadone; IV-MO, intravenous morphine; OR-MO, oral morphine; OR-OX, oral oxycodone; OS-ME, oral methadone; OTFC, oral transmucosal fentanyl.

assumption drawn from practical experience, suggesting a lack of relationship between the effective OTFC dose and fixed schedule of opioid regimen, regardless of the opioid used. ${ }^{6-9}$ However, observations from data pooled from these trials of OTFC showed a statistically significant relationship between the BTP dose and around-the-clock dose, despite an enormous interindividual variability in patients' dose requirements. ${ }^{11}$ Other studies with IV-MO have shown that IV-MO given as BTP medication in doses proportional to opioid basal regimen is safe and effective, ${ }^{4}$ and this observation was replied in a confirmatory study of a large number of episodes of BTP pain without evident risks even in aged population. ${ }^{5}$ As IV-MO has the highest potential risk for serious adverse event, one could argue that other drugs should be at least similarly safe. In a recent controlled study, similarly to IV-MO, OTFC used at a dose proportional to the basal opioid regimen was safe and effective in the majority of patients experiencing pain exacerbation. ${ }^{10}$

In this audit, reproducing a typical clinical scenario of an acute pain relief and palliative care unit, in which treatment is highly individualized, and different options are offered to patients according to the clinical need, opioids were given as BTP medication according to a construct based on the level of opioid tolerance provided by doses of opioids received for background analgesia. Thus, a dose proportional to the basal opioid regimen is given to counteract the increasing pain intensity. The intent of the study was not comparative, because opioids were freely chosen according to the clinical profile of each patient, and the aim was not to find the fastest and effective drug. For each patient, opioids were chosen according to individual needs, based on clinical judgment, including BTP presentation, type of opioid for background analgesia, patients' preference. For example, IV-ME or OR-ME were more frequently used in patients receiving methadone analgesia, oral route was used in patients with slow onset or nonsevere BTP intensity or simply because of patients' preference in respect to OTFC. Parenteral route or OTFC were chosen in patients who were unable to swallow or with nausea, or requiring prompt analgesia when more episodes occurred

TABLE 3. Percentage of Pain Reduction, According to the Opioid Administered

\begin{tabular}{rrrcccc}
\hline & IV-MO & OTFC & OR-MO & IV-ME & OR-ME & OX \\
\hline$<33 \%$ & 7 & 1 & 0 & 0 & 0 & 1 \\
$33-49 \%$ & 241 & 123 & 31 & 15 & 4 & 4 \\
$>50 \%$ & 225 & 121 & 31 & 13 & 3 & 4 \\
\hline
\end{tabular}

before achieving a stable basal dose. Although this group of patients should not be considered as having BTP, the treatment is otherwise the same. Thus, more often a compromise between clinical needs and patient preference was driving the decision. As expected IV-MO and OTFC, were the most frequent BTP medications used for BTP.

We also analyzed recently reported data according to the concept of cumulative response, ${ }^{13}$ which provide even more information about the number of patients with different percentage decreases in pain intensity in respect to earlier common cut-off of $33 \%$ and $50 \% .^{14}$ This study did not have comparative purposes and the results are obviously related to the choices dictated by the clinical judgment, rather than a comparison using homogeneous protocols. An individual treatment tailored according to the characteristics and preference of patients provided optimal outcome in a raw clinical scenario. The use of proportional doses provided a prompt response in most patients. The treatment was safe and effective, and only a minority of patients required further treatment. Drugs were given by trained nurses autonomously evaluating BTP and following the prescription as needed, ordered on the clinical sheet, according to the proportionality between the dose as needed and the background analgesics. A certain dose is necessary to cover the majority of BTP events with severe

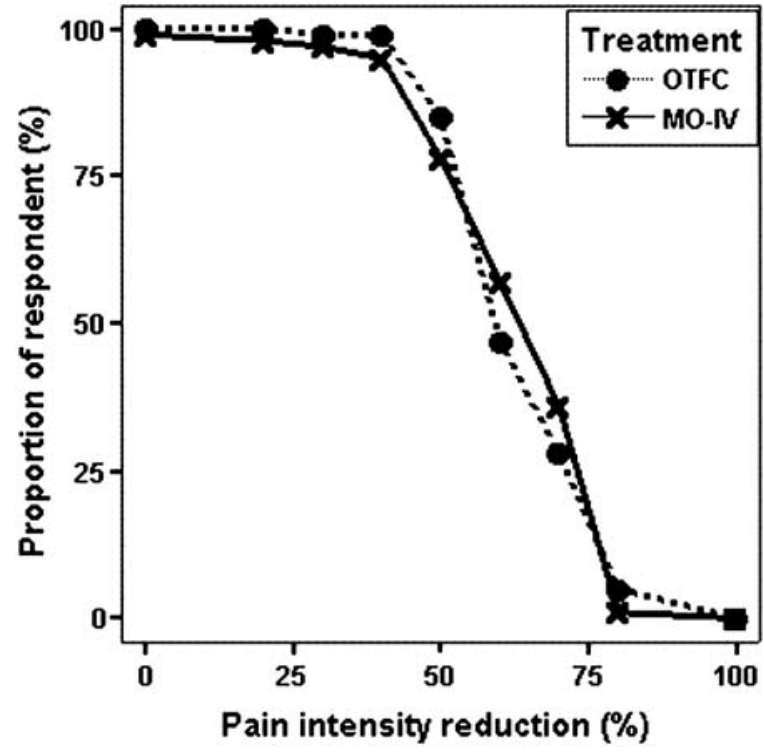

FIGURE 1. Cumulative proportion of responders with oral transmucosal fentanyl (OTFC) and intravenous morphine (IV-MO). 
intensity, which may have different presentations and unpredictable courses. It could be argued that this approach could expose patients to adverse effects. However, no patient developed severe adverse effects requiring a medical intervention, including older patients who could potentially be at risk, regardless of the type of opioid, dose, route, and age, confirming that tolerance is a protective factor against the occurrence of severe adverse effects, also explain the high percentage of successfully treated episodes. Of interest, oral medications were quite effective. This can be owing to the doses used ( $1 / 5$ of the opioid daily dose) or to a spontaneous pain relief, given that slow onset of any opioid administered orally.

In some way, the approach described refutes the need of titration, reported in earlier studies. ${ }^{6-9}$ This information, however, was not determined on the basis of a comparison between titration strategy and no titration strategy ${ }^{12}$ Despite an enormous interindividual variability, data pooled from the trials of the OTFC showed a statistically significant relationship between the BTP dose and aroundthe-clock dose. ${ }^{11}$ For instance, in an earlier open controlled study, IV-MO and OTFC in doses proportional to the scheduled daily dose of opioids were both safe and effective in patients experiencing pain exacerbation, ${ }^{10}$ suggesting that opioids given as needed could be safely used at proportional doses, regardless of the modality of administration. Preliminary and confirmatory surveys have shown the safety of this approach in a large number of patients with no life-threatening adverse effects occurring even in older patients. Respiratory depression, which is the most feared adverse effect, has never occurred, and no emergency call was needed. ${ }^{4,5}$ Finally, in a recent survey, reproducing a real clinical scenario, patients receiving a mean oral morphine dose of $132 \mathrm{mg}$, required $800 \mu \mathrm{g}$ of OTFC, ${ }^{15}$ suggesting that titration process may provide even higher doses than those expected by using proportional doses to basal opioid regimen.

The findings of this study suggest that in patients, receiving opioids for chronic cancer pain, the risks of administering doses of opioids proportional to the basal opioid regimen, even though relatively high, are minimal, either with opioids at fast or slow onset of action. This can be explained by the protective effect offered by opioid tolerance in patients chronically receiving relevant opioid doses for the management of the cancer pain.

For an appropriate evaluation of data presented, it should be taken into account that the setting of this observational study typically reflects the real clinical situation occurring in an acute palliative care unit, rather than that of a controlled study. The choice of opioids to be administered as needed was based on clinical and practical considerations, or patient's convenience; data gathered provide useful information about different modalities of opioid administration for BTP. The findings of this audit suggest that opioid doses for BTP could be based on the basal opioid regimen. This approach could prevent the need of titration that reduces patients' compliance. ${ }^{15,16}$ This approach cannot be generalized, owing to the characteristics of the unit, and this hypothesis should be tested in other settings and other contexts. Simplifying the treatment could help both patients and professionals. Controlled studies of different strategies to choice the opioid doses for treating BTP, for example, titration versus nontitration methods, should provide definitive information on this challenging issue.

\section{ACKNOWLEDGMENT}

The authors thank the nurse team for the invaluable efforts provided to collect data.

\section{REFERENCES}

1. Portenoy RK, Hagen NA. Breakthrough pain: definition, prevalence and characteristics. Pain. 1990;41:273-281.

2. Portenoy RK, Payne D, Jacobson P. Breakthrough pain: characteristics and impact in patients with cancer pain. Pain. 1999b;81:129-134.

3. Hanks GW, De Conno F, Cherny N, et al. Morphine and alternative opioids in cancer pain: the EAPC recommendations. Br J Cancer. 2001;84:587-593.

4. Mercadante S, Villari P, Ferrera P, et al. Safety and effectiveness of intravenous morphine for episodic-breakthrough pain, using a fixed ratio with the oral daily morphine dose. J Pain Symptom Manage. 2004;27:352-359.

5. Mercadante $S$, Intravaia $G$, Villari $P$, et al. Intravenous morphine for breakthrough (episodic-) pain in an acute palliative care unit: a confirmatory study. J Pain Symptom Manage. 2008;35:307-313.

6. Christie J, Simmonds M, Patt R, et al. Dose-titration multicenter study of transmucosal fentanyl citrate for the treatment of breakthrough pain in cancer patients using transdermal fentanyl for persistent pain. J Clin Oncol. 1998;16:3238-3245.

7. Farrar J, Cleary J, Rauck R, et al. Oral transmucosal fentanyl citrate: a randomized, double-blinded, placebo-controlled trial for treatment of breakthrough pain in cancer patients. $J$ Nat Cancer Inst. 1998;90:611-616.

8. Coluzzi P, Schwartzberg L, Conroy J, et al. Breakthrough cancer pain: a randomized trial comparing oral transmucosal fentanyl citrate (OTFC) and morphine sulphate immediate release (MSIR). Pain. 2001;91:123-130.

9. Portenoy RK, Payne R, Coluzzi P, et al. Oral transmucosal fentanyl citrate (OTFC) for the treatment of breakthrough pain in cancer patients: a controlled dose titration study. Pain. 1999a;79:303-312.

10. Mercadante S, Villari P, Ferrera P, et al. Transmucosal fentanyl versus intravenous morphine in doses proportional to basal opioid regimen for episodic-breakthrough pain. $\mathrm{Br} J$ Cancer. 2007;96:1828-1833.

11. Hagen NA, Fisher K, Victorino C, et al. A titration strategy is needed to manage breakthrough cancer pain effectively: observations from data pooled from three clinical trials. J Palliat Med. 2007;10:47-55.

12. Mercadante S. Breakthrough pain: on the road again. Eur J Pain. 2009;13:329-430.

13. Farrar JT, Dworkin RH, Max MB. Use of the cumulative proportion of responders analysis graph to present pain data over a range of cut-off points: making clinical trial data more understandable. J Pain Symptom Manage. 2006;31:369-377.

14. Farrar J, Portenoy RK, Berlin J, et al. Defining the clinically important difference in pain outcome measures. Pain. 2000; 88:287-294.

15. Zeppetella GB. Opioids for cancer breakthrough pain: a pilot study reporting patient assessment of time to meaningful pain relief. J Pain Symptom Manage. 2008;35:563-567.

16. Davies A, Vriens J, Kennett A, et al. An observational study of oncology patients' utilization of breakthrough pain medication. J Pain Symptom Manage. 2008;35:406-411. 